feet. In accord with the peat-like alluvium out of which they are excavated and constructed, their colour is a dark ashen grey or nearly black. A distinctive feature of these meridian ants' nests is their highly ornate architectural style. They consist of a congeries of slender pinnacles erected close to one another in the same straight line, and which are finally amalgamated. Upon these primary pinnacles numerous subsidiary ones are usuaily constructed. The completed edifice, with its full complement of spires and pinnacles, comes thus when viewed end on, as shown in certain of the photographs taken, to present in miniature a by no means remote resemblance to the architectural pile of some grand cathedral.

A second and simpler form of meridian ants' nest is especially abundant a few miles inland from Port Darwin. It differs from the preceding type in its more massive and less ornate structural plan. The upper edge is nearly smooth or irregularly serrated, but not produced into a series of slender pinnacles. It is further noteworthy, that the environments pertaining to these two meridian varieties differ materially. With the Port Darwin type the habitat affected is that of open grassy plains, while in the Laura Valley form the nest mounds are most abundantly constructed in typical, though thinly wooded forest land.

The raison dêtre of the north and south directions of the longer axial planes, so eminently characteristic of the so-called "Meridian" ants' nests, has given rise to much speculation and a variety of interpretations. By some it is supposed to bear a direct relationship to the prevailing winds. As, however, those in the districts where these ant-hills occur are chiefly south-east or north-west, according to the seasonal monsoon, that interpretation cannot be accepted as satisfactory. To the writer's mind a more probable explanation would appear to present itself in connection with the circumstance that being constructed in this precise meridian line, their larger surface presents the least possibly prolonged exposure to the meridianal ravs of the tropical sun, and that the structures are consequently so built that they shall absorb and retain a minimum amount of solar heat. This question is, however, an interesting one that undoubtedly invites further scientific investigation. The subject of Australian termitaria constitutes, it may be noted, a copiously illustrated chapter in the writer's recently published work, "The Naturalist in Australia."

W. SAVILLE-KENT.

\section{THE LIQUEFACTION OF FLUORINE.}

FLUORINE was prepared for the first time in I 886 by Prof. Moissan, as a product of the electrolysis of anhydrous hydrogen fluoride contained in a platinum apparatus provided with fluorspar stoppers; the new gas was at once found to be the most active chemical substance known, many elements and organic compounds, such as arsenic, antimony, sulphur, iodine, alcohol, and turpentine, immediately and spontaneously bursting into flame when plunged into an atmosphere of fluorine. On mixing the gas with hydrogen, even in the dark, a violent detonation immediately occurs; hydrogen fluoride being produced. The violent action of fluorine upon nearly all substances with which it is brought into contact, obviously renders extremely difficult all experimental work involving the use of the free element. The great manipulative difficulties necessarily arising whilst dealing with the gas on the large scale have, however, been very happily surmounted by Prof. Moissan and Prof. Dewar, who recently described to the Chemical Society the method by which they have succeeded in liquefying fluorine, and determining the more important properties of the liquid substance (Proc. Chem. Soc., November 4, 1897, p. 175). It seemed likely that the great chemical activity of fluorine might so far decrease at low temperatures as to allow of the manipulation of the material in a glass vessel cooled in liquid air ; this was found to be the case.

The fluorine required in the work was prepared by the electrolysis of anhydrous hydrogen fluoride; this liquid being a non-conductor, was made a conductor by dissolving in it potassium fluoride. The liberated fluorine was freed from hydrogen fluoride by being passed first through a platinum worm immersed in a cooling mixture of solid carbon dioxide and alcohol, and subsequently through platinum tubes containing dry sodium fluoride. The purified gas was then passed down a vertical platinum tube fused to the neck of a thin glass bulb which served as the collector, and an exit was provided through a narrower platinum tube contained inside the first. On cooling the apparatus down to $-183^{\circ}$ in boiling oxygen whilst the fluorine is passing through, no liquefaction occurs, but on reducing the pressure under which the oxygen is boiling, and so lowering the temperature to $-185^{\circ}$, the fluorine condenses in the glass bulb to a very mobile yellow liquid; on removing the bulb from the cooling bath the liquid fluorine boils vigorously. Other experiments made with boiling liquid oxygen and liquid air as refrigerating agents indicated that fluorine boils at about - $187^{\circ}$, namely at the boiling point of liquid argon; from this the probable critical temperature and pressure of fluorine are deduced as $-120^{\circ}$ and 40 atmospheres respectively.

At these low temperatures fluorine is without action on glass, and does not displace iodine from iodides; silicon, boron, carbon, sulphur, phosphorus and reduced iron, all of which spontaneously ignite when brought into contact with fluorine at ordinary temperatures, do not inflame if, after being cooled in liquid oxygen, they are plunged into an atmosphere of fluorine. Hydrogen gas inflames spontaneously, with considerable evolution of light and heat, when directed on to the surface of liquid fluorine at $-190^{\circ}$; on passing fluorine on to solidified turpentine cooled by boiling liquid air, a series of explosions occurred resulting in the destruction of the apparatus. It thus seems that the great affinity existing between hydrogen and fluorine is not overcome at - I90. A little liquid fluorine falling on the floor instantly inflames the wood. Fluorine is soluble in liquid oxygen, and on passing in the gas a white flocculent precipitate is formed which, after filtering off, deflagrates violently as the temperature rises; it is possibly a hydrate of fluorine.

Determinations made by floating pieces of various substances in liquid fluorine indicate that its density is about I.I4, and from the invisibility of amber immersed in the liquid the refractive index of the latter would seem to be higher than that of liquid air or oxygen. Liquid fluorine shows no magnetic phenomena when placed between the poles of a powerful electromagnet ; it has a smaller capillarity constant than liquid oxygen, and does not solidify at $-210^{\circ}$. It has no absorption spectrum, and its colour is the same as that of the gaseous element.

W. J. P.

\section{THE LEONID DISPLAY, 1897.}

VERY unfavourable weather appears to have prevented the successful observation of the Leonids at their recent return. In consequence of this the impression seems to have gained ground that the phenomenon did not occur as predicted. This is, however, a mistake. Could those observers who saw so little on the night of the 13 th, have viewed the sky late on the following night, they must have been satisfied at the character of the display. Between about 4.30 and 6 a.m. on Monday morning the 15 th, the usual streak-leaving meteors from Leo became very numerous, and some of them were unusually brilliant, one, which

No. $146_{5}$, VOL. 57$]$ 